\title{
A Three-Axis Single-Proof-Mass CMOS-MEMS Piezoresistive Accelerometer with Frequency Output
}

\author{
Yi Chiu*, Tsung-Chih Huang and Hao-Chiao Hong \\ Department of Electrical and Computer Engineering, National Chiao Tung University \\ Hsin Chu 300, Taiwan, R.O.C.
}

(Received June 10, 2013; accepted October 8, 2013)

Key words: $\quad$ accelerometer, CMOS MEMS, piezoresistive, frequency output, RC oscillator

Accelerometers have been used in a wide range of applications such as automobiles, mobile phones, and game controllers. In this paper, we present a monolithic complementary metal-oxide-semiconductor micro-electromechanical systems (CMOSMEMS) accelerometer with frequency output. The output oscillation frequency can be converted to a digital code by using a counter so that the sensor can be easily integrated with digital signal processing units on the same chip. The accelerometer is composed of a single proof mass suspended by four suspension beams. Polysilicon piezoresistors are placed at the ends of the beams to sense the displacement of the proof mass. The piezoresistors are used in RC oscillators whose oscillation frequencies vary owing to the change in resistance upon application of an external acceleration. Acceleration components in three axes can be obtained by the proper combination of signals from the piezoresistors at different locations. The accelerometer was fabricated by standard CMOS processes followed by backside and frontside dry etching postprocessing. The measured mechanical resonant frequency is $464 \mathrm{~Hz}$. The oscillation frequency of the $z$-axis oscillator is about $70 \mathrm{MHz}$. The measured absolute sensitivity, relative sensitivity, and resolution along the $z$-axis are $198 \mathrm{kHz} / g, 2.8 \times 10^{-3} \Delta f / f_{0} / g$, and $10.9 \mathrm{mG} / \sqrt{\mathrm{Hz}}$, respectively, for a sampling rate of $400 \mathrm{~Hz}$ and acceleration of $6 \mathrm{~g}$ at $27 \mathrm{~Hz}$.

\section{Introduction}

Accelerometers are currently the most popular micro-electromechanical systems (MEMS) sensors in the market. They have been used in various applications such as airbag sensors in automobiles, inertial sensors in navigation systems, and motion sensors in game controllers and mobile phones. According to the sensing principles, the MEMS accelerometers can be capacitive, piezoresistive, or thermal devices. In capacitive

*Corresponding author: e-mail: yichiu@mail.nctu.edu.tw 
accelerometers, ${ }^{(1-3)}$ the acceleration is sensed by detecting the change in variable sensing capacitance. The advantages of the capacitive accelerometers include high speed and low power consumption. However, the fabricated sensing capacitor can be deformed owing to the residual stress in the mechanical structures. This causes a significant reduction of sensitivity or mismatch in the input that can affect the functionality of the sensing circuits. Thermal accelerometers detect acceleration by sensing the change in temperature of the sensing elements owing to heated moving fluid. ${ }^{(4,5)}$ In general, they consume more power and have a lower response speed. Piezoresisitve accelerometers detect the acceleration by sensing the resistance change caused by the structural deformation attributable to external acceleration. ${ }^{(6-10)}$ In comparison, they have less power consumption and simpler sensing circuits. The mechanical structures are also simpler and less sensitive to the residual stress than those of the capacitive accelerometers; thus, the yield can be improved and the manufacturing cost can be reduced. Even though the sensing resistance can be affected by temperature variation, proper circuit or sensor compensation can be used to reduce the temperature effect. ${ }^{(6,11-13)}$

Accelerometers can also be classified as analog or digital according to their output formats. Most of the MEMS accelerometers use C-V converters or Wheatstone bridges to obtain analog output signals. Offset and mismatch of circuit elements can cause circuit saturation and reduction of dynamic range in these direct sensing circuits. Therefore, special techniques such as correlated double sampling or chopper stabilization must be used to avoid the offset or parasitic effects. The analog output signals are converted to digital codes by using analog-to-digital converters (ADCs). However, high-resolution ADCs are difficult to design and require more chip area. In comparison, sensors with frequency output have the advantage of less sensitivity to interference and noise, wide oscillation range and thus large signal dynamic range, and tunable bandwidth and resolution. The output frequency can be converted to a digital code by a counter that is much easier to design and more robust than the ADC.

In frequency-output accelerometers, the shift of the output oscillation frequency $\Delta f$ is proportional to the center frequency $f_{0}$. If a counter is used to convert the output frequency to a digital code, large center frequency $f_{0}$ and frequency shift $\Delta f$ are both desired to reduce the readout time to resolve the frequency shift. Most of the demonstrated MEMS frequency-output accelerometers use stress-loaded micromechanical resonators as the frequency-selecting elements. They can be electrostatically driven with capacitive sensing ${ }^{(14-16)}$ or electrothermally driven with piezoresistive sensing. ${ }^{(17-19)}$ Whereas mechanical oscillators have high quality factors (Q) and thus good stability and noise performance, their oscillation frequencies, which are usually in the range of 2 to $200 \mathrm{kHz}$, are relatively low owing to their mechanical nature. The mechanical oscillators need vacuum packaging to reduce the Brownian noise and achieve high Q. This special packaging requirement increases the total manufacturing cost. In contrast, the electricaloscillator-based accelerometers can achieve an oscillation frequency above GHz. ${ }^{20,21)}$ For example, a complementary metal-oxide-semiconductor MEMS (CMOS-MEMS) LC oscillator was used in an accelerometer with a center frequency of around $2 \mathrm{GHz} .{ }^{(21)}$ It had a much higher sensitivity of $3.62 \mathrm{MHz} / g$, compared with 2 to $500 \mathrm{~Hz} / \mathrm{g}$ for typical mechanical-oscillator-based accelerometers. Another advantage of electrical-oscillatorbased accelerometers is that they do not require the driving mechanism as is required 
for the mechanical oscillators. Thus, the system design can be simplified. Even though the $\mathrm{Q}$ factors of electrical oscillators are inferior to those of the mechanical oscillators, they can achieve sub-ppm stability without vacuum packaging if properly designed. ${ }^{(22)}$ Therefore, they are still attractive for applications such as automobiles and consumer electronics where cost is essential and the performance requirement is less stringent.

A piezoresistive RC relaxation oscillator was demonstrated in a MEMS inclinometer/ accelerometer composed of discrete sensor elements, signal conditioning ICs, and other electronic components. ${ }^{(23)}$ The center frequency and sensitivity were $4.6 \mathrm{kHz}$ and 148 $\mathrm{Hz} / \mathrm{g}$, respectively. The low center frequency was probably caused by the use of external components. In this paper, we demonstrate a monolithic RC-oscillator-based CMOSMEMS piezoresistive accelerometer with high center frequency and large sensitivity. A single proof mass was suspended by four suspension beams in which piezoresistors were embedded to sense acceleration-induced proof mass displacement. An RC oscillator was used to convert the resistance change to a frequency shift. The device was first fabricated by standard $0.35 \mu \mathrm{m}$ CMOS processes. Dry etching post-CMOS processes were then used to define the suspension beams and release the proof mass. The design, fabrication, and characterization of the proposed accelerometer are presented in the following sections.

\section{Principle}

\subsection{Operation principle}

In the proposed accelerometer, the sensing piezoresistors $R$ are embedded in the suspension beams of the proof mass. The resistors are connected with a capacitor $C$ to form the core of a piezoresistive $\mathrm{RC}$ oscillator whose oscillation frequency $f$ is proportional to $1 / R C$. The displacement of the proof mass attributable to the external acceleration $a$ causes strain and stress in the suspension beams and induces a change in resistance $\Delta R$, which can be calculated from the piezoresistive effect,

$$
\frac{\Delta R}{R_{0}}=G \frac{\sigma}{E}
$$

where $R_{0}$ is the resistance without the acceleration, $G$ is the gauge factor (approximately 30 for polysilicon), $E$ is the Young's modulus (approximately 160 $\mathrm{GPa}$ for polysilicon), and $\sigma$ is the stress in the resistor, which is proportional to the acceleration. Upon application of the acceleration, the oscillation frequency becomes $f=f_{0}+\Delta f \propto f_{0}\left(1+\Delta R / R_{0}\right)$. Thus, the frequency shift $\Delta f$ is proportional to the acceleration $a$ and center frequency $f_{0}$. Compared with mechanical oscillators, the RC oscillators can achieve a much higher center frequency $f_{0}$. Therefore, the frequency shift $\Delta f$ can be enhanced using electrical RC oscillators. Large $f_{0}$ and $\Delta f$ are both desired since they reduce the counter readout time and thus increase the signal bandwidth.

The block diagram of the accelerometer is shown in Fig. 1. The oscillator has two piezoresistors $R_{\mathrm{p}}$ and $R_{\mathrm{n}}$. The acceleration induces a differential change in resistance in the two resistors, i.e., 


$$
R_{\mathrm{p}, \mathrm{n}}=R_{0} \pm \Delta R
$$

A counter is used to convert the output frequency $f_{0}+\Delta f$ of the oscillator to a digital code at a sampling frequency $f_{\mathrm{s}}$. The output signal bandwidth and resolution can be controlled by adjusting $f_{\mathrm{s}}$. The schematic of the piezoresistive RC oscillator is shown in Fig. 2. The two piezoresistors $R_{\mathrm{p}, \mathrm{n}}$ in Fig. 1 are connected to a constant voltage source $V_{\mathrm{R}}$ to generate two current sources $I_{\mathrm{p}, \mathrm{n}}=V_{\mathrm{R}} / R_{\mathrm{p}, \mathrm{n}}$. The current sources are controlled by a Schmitt trigger to charge and discharge the capacitor $C$ alternately, producing a steady oscillation output. When the Schmitt trigger output $V_{\mathrm{o}}$ is high, the difference current $I_{\mathrm{C}}=I_{\mathrm{p}}-I_{\mathrm{n}}$ charges the capacitor and the capacitor voltage $V_{\mathrm{C}}$ increases linearly with time. When $V_{\mathrm{C}}$ reaches the high threshold $V_{\mathrm{OH}}$, the Schmitt trigger output becomes low and the charging current is reversed to discharge the capacitor. Thus, $V_{\mathrm{C}}$ decreases linearly with time, as shown in Fig. 2. When $V_{\mathrm{C}}$ reaches the low threshold $V_{\mathrm{OL}}$, the output of the Schmitt trigger becomes high again to charge the capacitor. The charging-discharging cycles repeat periodically to produce the output oscillation signals.

The oscillation frequency is proportional to the charging/discharging current $I_{\mathrm{C}}=\left|I_{\mathrm{p}}-I_{\mathrm{n}}\right|$. The current sources $I_{\mathrm{p}}$ and $I_{\mathrm{n}}$ are multiplied by factors of 5 and 6 , respectively, so that

$$
I_{\mathrm{C}}=\left|I_{\mathrm{p}}-I_{\mathrm{n}}\right|=6 \frac{V_{\mathrm{R}}}{R_{\mathrm{n}}}-5 \frac{V_{\mathrm{R}}}{R_{\mathrm{p}}} .
$$

Therefore, the charging current has a nonzero bias to sustain the oscillation even when $R_{\mathrm{n}}$ $=R_{\mathrm{p}}$ in the absence of acceleration. From eqs. (2) and (3),

$$
I_{\mathrm{C}}=V_{\mathrm{R}}\left(\frac{6}{R_{0}-\Delta R}-\frac{5}{R_{0}+\Delta R}\right)=V_{\mathrm{R}}\left(\frac{R_{0}+11 \Delta R}{R_{0}^{2}-(\Delta R)^{2}}\right) \approx \frac{V_{\mathrm{R}}}{R_{0}}\left(1+\frac{11 \Delta R}{R_{0}}\right) .
$$

The frequency of the charging/discharging oscillation, as shown in Fig. 2, is

$$
f=f_{0}+\Delta f=\frac{I_{\mathrm{C}}}{C} \frac{1}{2\left(V_{\mathrm{OH}}-V_{\mathrm{OL}}\right)}=\frac{V_{\mathrm{R}}}{2 C R_{0}\left(V_{\mathrm{OH}}-V_{\mathrm{OL}}\right)}\left(1+\frac{11 \Delta R}{R_{0}}\right)=f_{0}\left(1+\frac{11 \Delta R}{R_{0}}\right) .
$$
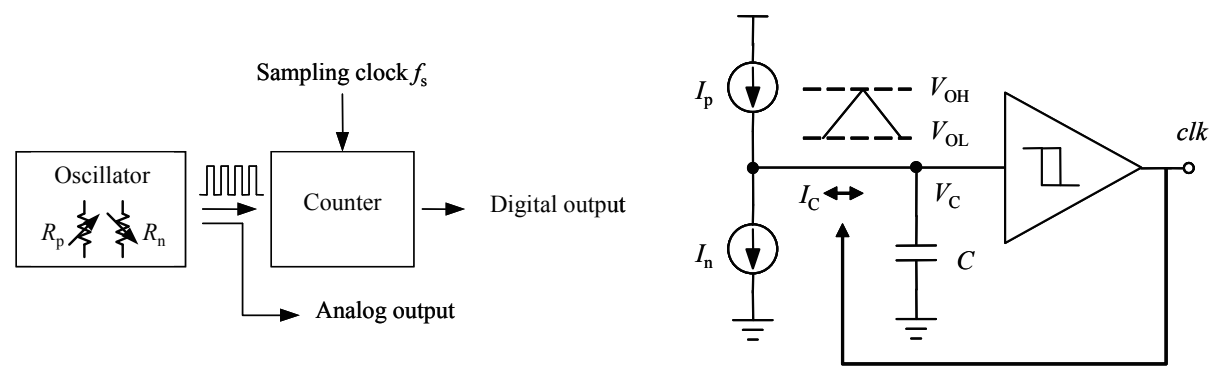

Fig. 1 (left). Block diagram of proposed accelerometer.

Fig. 2 (right). Schematic of piezoresistive RC oscillator. 
Thus, $\Delta f \propto \Delta R \propto a$ and the acceleration $a$ can be determined by measuring the frequency shift $\Delta f$. The second term in eq. (5) shows that this design can enhance the sensitivity of the accelerometer by a factor of 11 .

\subsection{MEMS sensor}

Figure 3 shows the mechanical structure of the MEMS sensor. The center proof mass is fabricated by etching the substrate of a CMOS chip to increase its thickness and mass. The silicon proof mass is suspended by four beams composed of the oxide, metal, and polysilicon layers in the standard CMOS processes. Polysilicon piezoresistors are placed at the two ends of the beam, where the stress is maximum when deformed, to sense the acceleration-induced displacement of the mass. As shown in Fig. 3(a), each suspension beam has two embedded sensing resistors near the bottom surface of the beam: the a-resistor is close to the connection between the beam and the proof mass, whereas the b-resistor is close to the connection between the beam and the CMOS substrate. When the fixed-guided beam deforms, the two resistors are subjected to stress with equal magnitude but opposite signs, i.e., $R_{\mathrm{a}, \mathrm{b}}=R_{0} \pm \Delta R$.

When the accelerometer is subjected to a $z$-axis acceleration, the mass moves vertically and the resistance change of the eight piezoresistors has the following relationship,

$$
\begin{gathered}
\Delta R_{\mathrm{a} 1}=\Delta R_{\mathrm{a} 2}=\Delta R_{\mathrm{a} 3}=\Delta R_{\mathrm{a} 4}=\Delta R, \\
\Delta R_{\mathrm{b} 1}=\Delta R_{\mathrm{b} 2}=\Delta R_{\mathrm{b} 3}=\Delta R_{\mathrm{b} 4}=-\Delta R .
\end{gathered}
$$

To sense the $z$-axis acceleration, these resistors are electrically connected in series to form two differential resistors,

$$
\begin{aligned}
& R_{\mathrm{p} z}=R_{\mathrm{a} 1}+R_{\mathrm{a} 2}+R_{\mathrm{a} 3}+R_{\mathrm{a} 4}, \\
& R_{\mathrm{n} z}=R_{\mathrm{b} 1}+R_{\mathrm{b} 2}+R_{\mathrm{b} 3}+R_{\mathrm{b} 4} .
\end{aligned}
$$

with $R_{\mathrm{p} z \mathrm{nz}}=4 R_{0} \pm 4 \Delta R$. When the accelerometer is subject to an $x$-axis acceleration as defined in Fig. 3(a), the mass rotates about the $y$-axis. In this case, the resistance change of the eight piezoresistors has the following relationship,

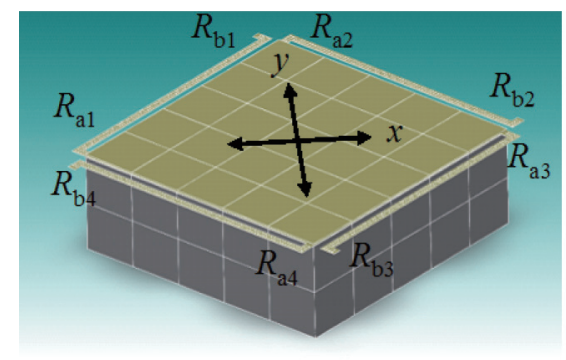

(a)

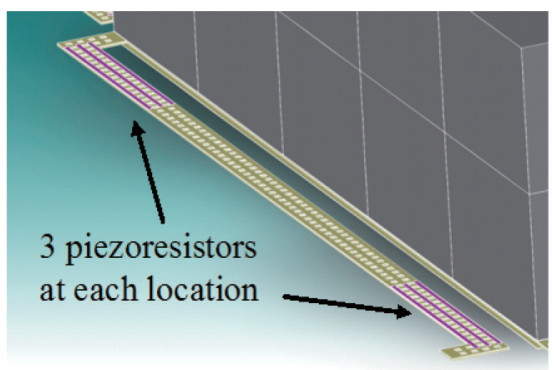

(b)

Fig. 3. (Color online) Schematic of suspended proof mass. 


$$
\begin{gathered}
\Delta R_{\mathrm{a} 1}=\Delta R_{\mathrm{b} 2}=\Delta R_{\mathrm{b} 3}=\Delta R_{\mathrm{a} 4}=\Delta R, \\
\Delta R_{\mathrm{b} 1}=\Delta R_{\mathrm{a} 2}=\Delta R_{\mathrm{a} 3}=\Delta R_{\mathrm{b} 4}=-\Delta R .
\end{gathered}
$$

Similar to the $z$-axis sensing, these resistors are connected to form two differential resistors,

$$
\begin{aligned}
& R_{\mathrm{p} x}=R_{\mathrm{a} 1}+R_{\mathrm{b} 2}+R_{\mathrm{b} 3}+R_{\mathrm{a} 4}, \\
& R_{\mathrm{n} x}=R_{\mathrm{b} 1}+R_{\mathrm{a} 2}+R_{\mathrm{a} 3}+R_{\mathrm{b} 4} .
\end{aligned}
$$

with $R_{\mathrm{p} x, \mathrm{n} x}=4 R_{0} \pm 4 \Delta R$ to sense the $x$-axis acceleration. The same principle can be applied to the combination of resistors for the $y$-axis acceleration sensing. Physically, three identical resistors are placed at each location in the beam, as shown in Fig. 3(b), so that three sets of differential resistors can be wired independently to sense the acceleration in three axes.

This wiring scheme can also eliminate the cross-axis sensitivity in this single-mass accelerometer. For example, a $z$-axis acceleration causes the resistance change in the sensing resistors $R_{\mathrm{p} z, \mathrm{nz}}$ according to eq. (6). If eq. (6) is substituted into eqs. (8) and (9), it can be seen that $R_{\mathrm{p} x, \mathrm{nx}}=4 R_{0} \pm(\Delta R-\Delta R-\Delta R+\Delta R)=4 R_{0}$. Therefore, there is no change in resistance in the $x$-axis sensing resistors and thus no frequency shift in the $x$-axis oscillator.

\subsection{CMOS oscillator circuit}

Figure 4 shows the operation of the oscillator in different phases. The direction of current flow of the two current sources $I_{\mathrm{p}}$ and $I_{\mathrm{n}}$ is controlled by manipulating the control signal clk. In the charging phase [Fig. 4(a)], the current control signal clk is high and the difference current $I_{\mathrm{C}}$ flows into the capacitor to charge it. In the discharging phase, the control signal $c l k$ is low and $I_{\mathrm{C}}$ is reversed so that it flows out of the capacitor to discharge it. The sensing resistor is composed of four $3-\mathrm{k} \Omega$ resistors in series, as discussed in the previous section. To reduce the thermal noise on the resistors, the current is limited to $100 \mu \mathrm{A}$ and thus the voltage $V_{\mathrm{R}}$ is set to $1.2 \mathrm{~V}$. As shown in Fig. 4, there are three metal-oxide-semiconductor field-effect-transistors (MOSFETs) cascaded between the supply voltage $V_{\mathrm{DD}}$ and the capacitor $C$ and between the capacitor and the GND. To ensure the proper operation of the current sources, the voltage switching threshold $V_{\mathrm{OH}}-V_{\mathrm{OL}}$ is set to $0.5 \mathrm{~V}$. The capacitor is set to $1 \mathrm{pF}$ so that the nominal oscillation frequency is $100 \mathrm{MHz}$, according to eq. (5). A buffer was used after the Schmitt trigger to generate a rail-to-rail output square wave.

\section{Design and Simulation}

\subsection{MEMS design}

The sensor and circuits were implemented by using standard $0.35 \mu \mathrm{m} 2 \mathrm{P} 4 \mathrm{M}$ CMOS processes. Owing to the chip area limit, the maximum area of the suspended proof mass was set to $1 \times 1 \mathrm{~mm}^{2}$. The thickness of the proof mass was $300 \mu \mathrm{m}$ to reduce the time 


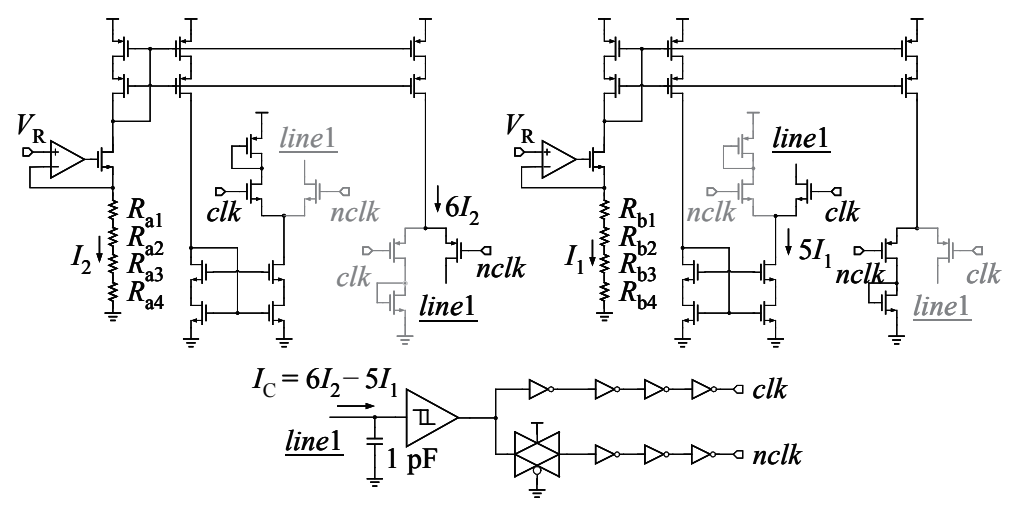

(a)

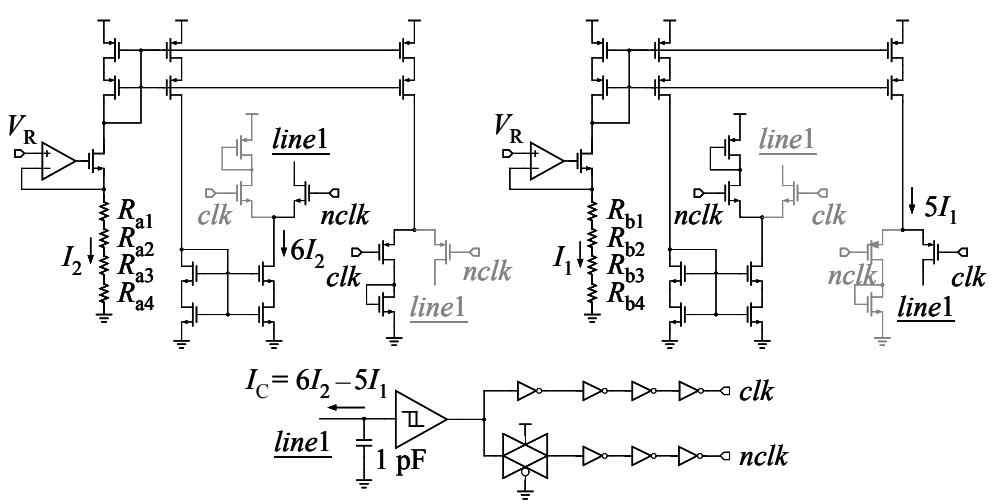

(b)

Fig. 4. (a) Charging and (b) discharging phases of $z$-axis oscillator.

and cost of the postprocesses. The suspension beams were composed of the layers from M2 to field oxide with a total thickness of $2.93 \mu \mathrm{m}$. The width and length of the beams were $32 \mu \mathrm{m}$ and $1 \mathrm{~mm}$, respectively. Two devices were designed with silicon proof mass areas of $1 \times 1 \mathrm{~mm}^{2}$ (device I) and $0.25 \times 0.25 \mathrm{~mm}^{2}$ (device II). The mechanical resonance frequencies of the fundamental modes of devices I and II simulated by Coventorware were 87 and $389 \mathrm{~Hz}$, respectively, as shown in Fig. 5. Figure 6 shows the stress simulation in the suspension beams of device I under $1 \mathrm{~g}$ acceleration in the $z$-direction. The maximum stress and the corresponding resistance change $\Delta R / R_{0}$ were about $50 \mathrm{MPa}$ and $1 \%$, respectively. A similar simulation was also performed for device II. Owing to the smaller mass, the maximum stress and the corresponding resistance change $\Delta R / R_{0}$ were about $2.5 \mathrm{MPa}$ and $0.05 \%$, respectively, under $1 \mathrm{~g}$ acceleration.

\subsection{Circuit design}

Without acceleration $\left(\Delta R / R_{0}=0\right)$, the prelayout Hspice simulation results of the oscillator in Fig. 4 are shown in Fig. 7. The oscillation frequency was about $97 \mathrm{MHz}$, 


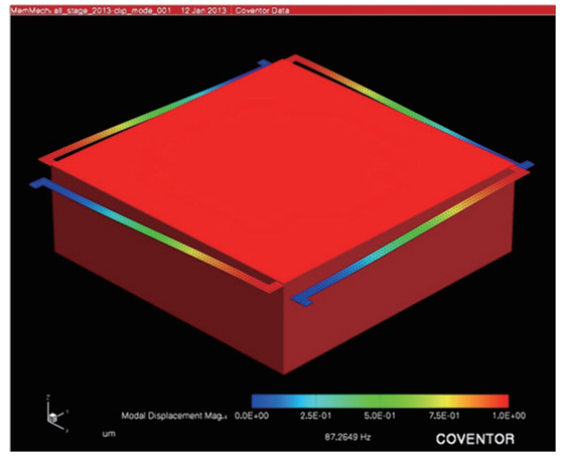

(a)

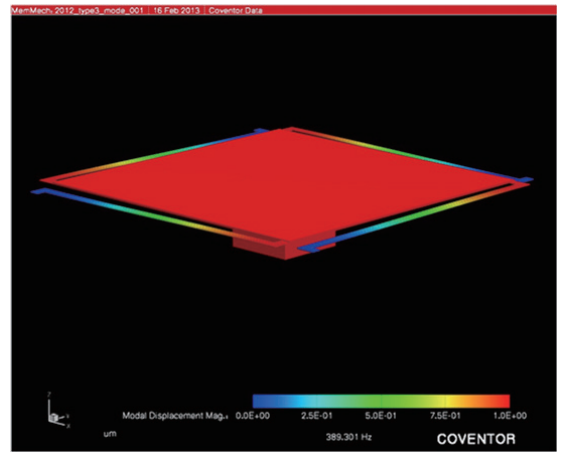

(b)

Fig. 5. (Color online) Coventorware simulation of fundamental resonance modes, (a) device I and (b) device II.

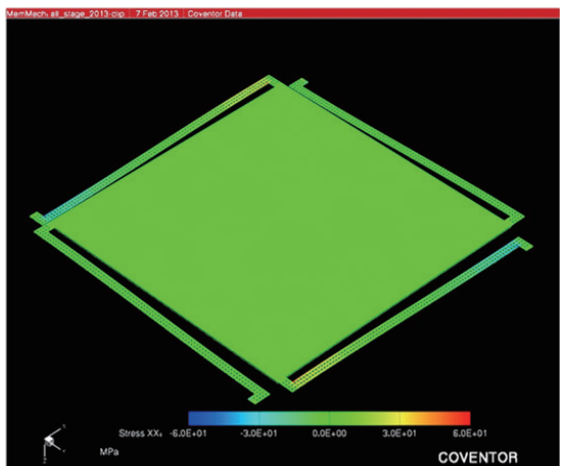

(a)

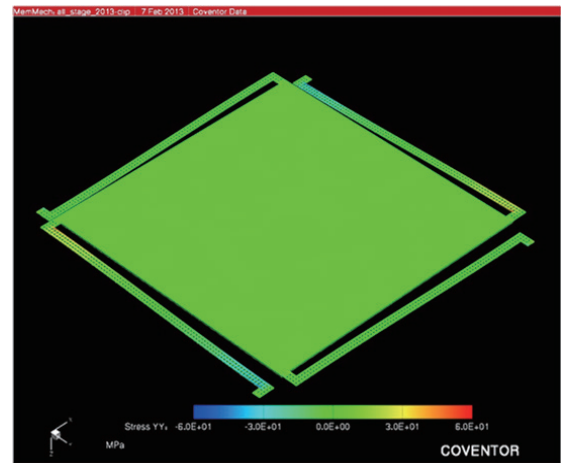

(b)

Fig. 6. (Color online) Coventorware simulation of stress in suspension beams under $1 \mathrm{~g}$ acceleration in $z$-direction, (a) $\sigma_{x x}$ and (b) $\sigma_{y y}$.

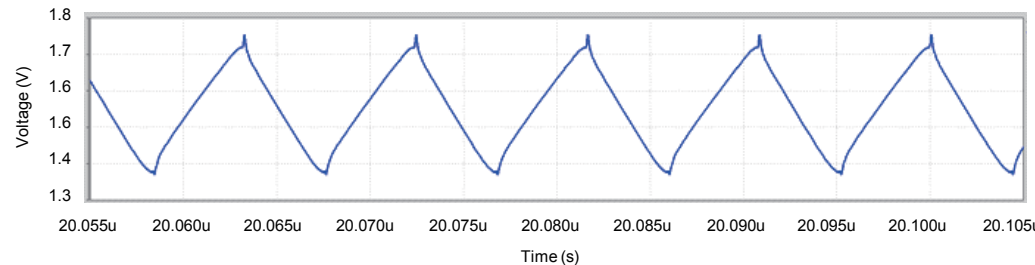

(a)

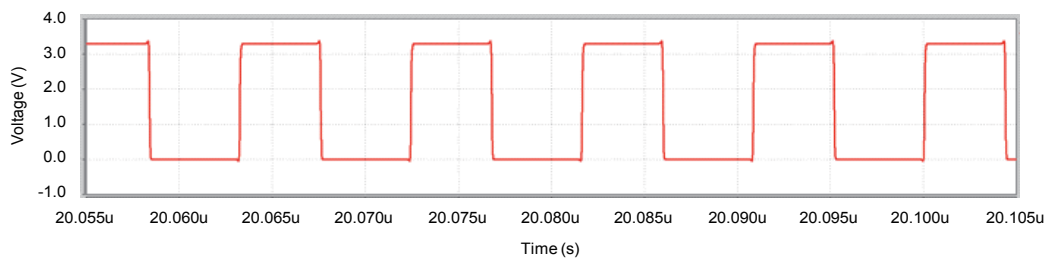

(b)

Fig. 7. (Color online) Simulated output waveforms of oscillator, (a) voltage on capacitor $C$ and (b) voltage of Schmitt trigger output. 
close to the theoretical calculation. In the postlayout simulation, the parasitic capacitance and resistance effects were taken into consideration, and the oscillation frequency was lowered to about $94 \mathrm{MHz}$. The frequency shift attributable to the resistance change $\left|\Delta R / R_{0}\right|$ up to $1 \%$, corresponding to an external acceleration of $\pm 1 \mathrm{~g}$ for device $\mathrm{I}$, is shown in Fig. 8 for various process corners. The simulation results show a consistent absolute sensitivity of $6.8 \mathrm{MHz} / g$ and relative sensitivity of $7.2 \% \Delta f / f_{0} / g$. The center frequency variation for various corners observed in the simulation can be calibrated in the testing phase and will not affect the accelerometer performance in practice. For device II, the reduced mass caused a reduction of sensitivity, which could be estimated from the simulated mechanical response to be $340 \mathrm{kHz} / g$ and $3.6 \times 10^{-3} \Delta f / f_{0} / g$.

\section{CMOS-MEMS Postprocessing}

The CMOS chips from the foundry must be processed to release the proof mass for the accelerometer to function. The post-CMOS processes are shown in Fig. 9. First, the chip was thinned to a thickness of $300 \mu \mathrm{m}$ to reduce the time and cost of the subsequent deep reactive ion etching (DRIE) step [Fig. 9(b)]. By reducing the etching time and thus the heat dissipation problem, the yield of the etching process can be improved. Then, a 4.5- $\mu \mathrm{m}$-thick AZ 4620 photoresist was applied to the backside of the chip and patterned to define the proof mass area [Fig. 9(c)]. A DRIE process was used to etch through the 300- $\mu \mathrm{m}$-thick substrate to define the proof mass structure. After the DRIE step, a spacer was attached to the backside of the chip to protect the bottom surface of the proof mass during handling [Fig. 9(d)]. Finally, frontside reactive ion etching (RIE) was used to etch the $\mathrm{SiO}_{2}$ layer and define the suspension beams by using the M2 layer as the etching mask. Upon completion of the final etching step, the suspended proof mass is released. The released accelerometer is rather fragile owing to the large mass and thin suspension beams. Only device II with a smaller proof mass $\left(0.25 \times 0.25 \mathrm{~mm}^{2}\right)$ survived the postprocessing and wire bonding steps. As shown in Fig. 10, the residual stress in the oxide-metal layers of the CMOS processes caused bending of the suspension beams and diaphragm. Nevertheless, the shape of the rigid proof mass can still be seen in the figure.

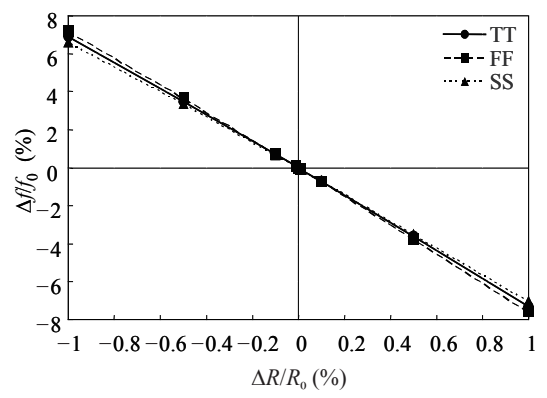

Fig. 8. Postlayout simulation of relative frequency shift for various process corners. 


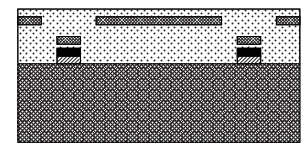

(a) as-received chip

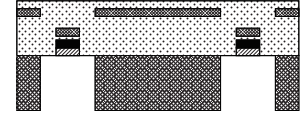

(c) backside photolithography and etching

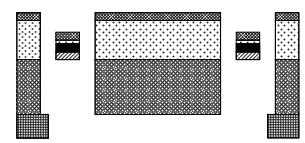

(e) frontside etching and release

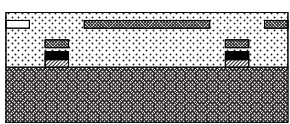

(b) backside thinning

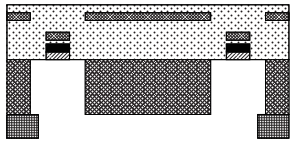

(d) attaching spacer
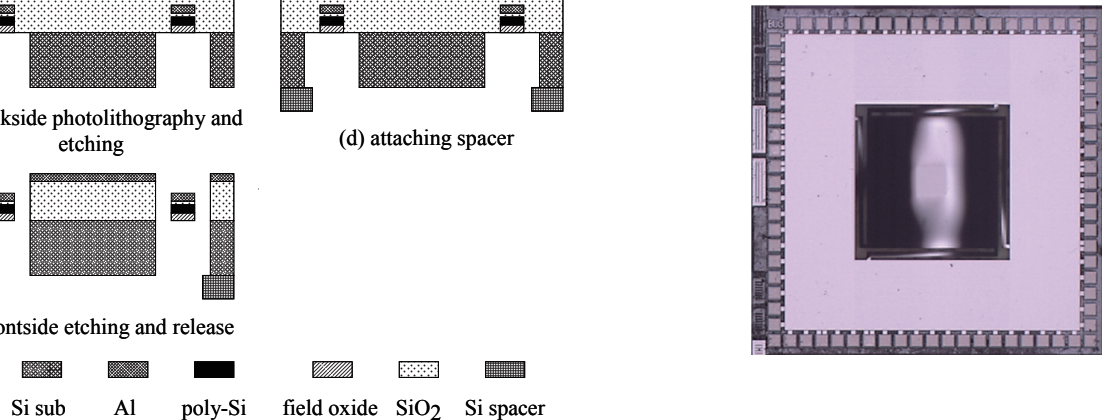

Fig. 9 (left). Post-CMOS processes.

Fig. 10 (right). (Color online) Device II, after post-CMOS processing.

\section{Measurement and Discussion}

\subsection{Mechanical measurement}

A laser Doppler vibrometer (LDV) was used to measure the mechanical resonance frequency of the released device. Figure 11 shows that device II has a resonance frequency of $464 \mathrm{~Hz}$, close to the simulation value of $389 \mathrm{~Hz}$. The possible causes of this discrepancy include variation in the pattern and thickness of the proof mass, material properties, and residual stress in the suspension beams and diaphragm. The quality factor calculated from the width of the resonance peak is about 4.6. This relatively small quality factor is possibly caused by the large area of the suspension diaphragm that can cause significant damping in the vertical motion.

\subsection{Oscillator measurement}

The oscillator output, i.e., the clk signal in Fig. 4, was observed using an oscilloscope. Figure 12 shows the output waveform of the $y$-axis oscillator. The oscillation frequency is about 95.5 MHz. Similar measurement shows that the frequencies of the $x$ - and $z$-axis oscillators are 73.2 and $70.3 \mathrm{MHz}$, respectively. The main reason for the frequency difference is that the Schmitt triggers in the $x$ - and $z$-axis oscillators are based on operational amplifiers (OPs), whereas the $y$-axis oscillator has a digital Schmitt trigger in the feedback loops. Compared with the OP-based trigger, the digital Schmitt trigger has a larger bandwidth, thus allowing a higher oscillation frequency. An Agilent E4440A spectrum analyzer was used to characterize the phase noise in the oscillator output, as shown in Fig. 13. The phase noise at $1 \mathrm{MHz}$ frequency offset is about $90 \mathrm{dBc} / \mathrm{Hz}$, comparable to the performance of typical RC oscillators. ${ }^{(24)}$ 


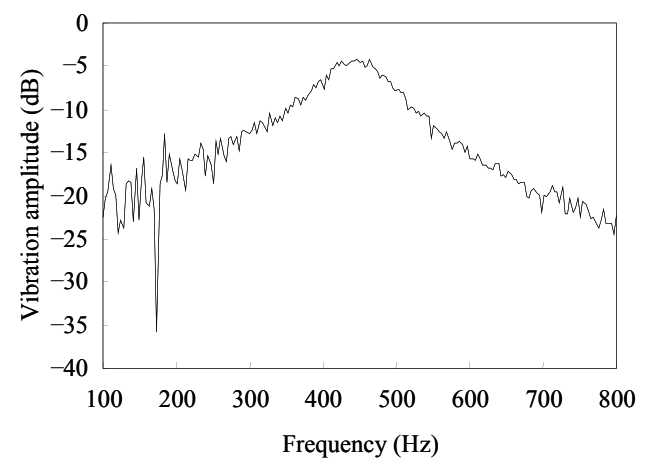

Fig. 11. LDV measurement of first resonance mode of device II.

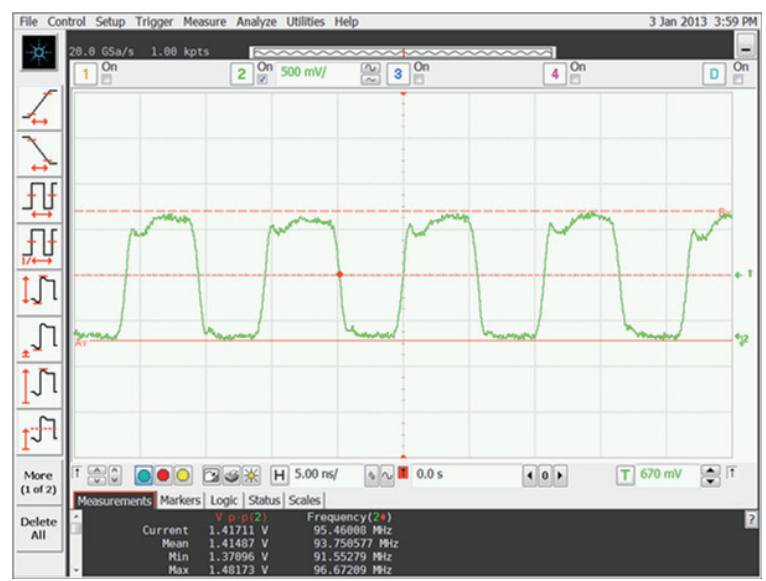

Fig. 12. (Color online) Oscillation waveform of $y$-axis oscillator.

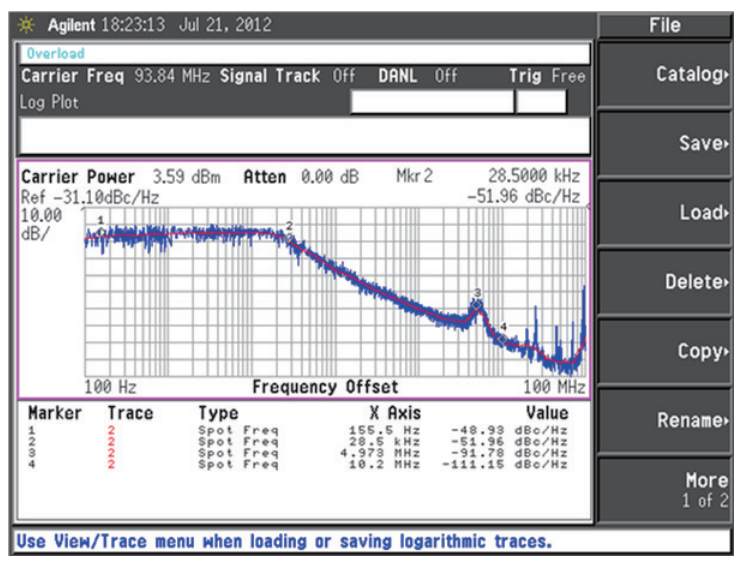

Fig. 13. (Color online) Phase noise of $y$-axis oscillator. 


\subsection{Acceleration test}

The $x$ - and $y$-axis sensing circuits were damaged during testing. Therefore, only the $z$-axis accelerometer was used in the dynamic acceleration testing. The accelerometer was mounted on an LDS V406 shaker that generated an acceleration with amplitude $g_{\text {in }}$ at vibration frequency $f_{\text {in }}$. A commercial accelerometer (PCB Piezotronics model 353B17) was used to calibrate the vibration levels. The output of the oscillator was connected to a Stanford SR620 frequency counter to measure the oscillation frequency. An Agilent 33250A function generator was used to generate the sampling clock for the counter at sampling frequency $f_{\mathrm{s}}$. The counter output was collected by a computer for further analysis. Figure 14 shows the accelerometer output signal for $f_{\text {in }}=27 \mathrm{~Hz}$, $f_{\mathrm{s}}=400 \mathrm{~Hz}$, and $g_{\text {in }}=3 \mathrm{~g}$. The sensitivity calculated from curve fitting is $198 \mathrm{kHz} /$ g. The fast Fourier transform (FFT) analysis of Fig. 14 is shown in Fig. 15. Owing to the large flicker noise at low frequency, the signal-to-noise ratio (SNR) calculated from the FFT spectrum is $25.85 \mathrm{~dB}$, corresponding to a resolution of $10.9 \mathrm{mg} / \sqrt{\mathrm{Hz}}$. It can also be seen that the output has relatively large harmonic components, which are probably caused by the distortion of the excitation shaker input and the asymmetry of the mechanical sensing structures. Figure 16 shows the sensor performance at $f_{\text {in }}=27 \mathrm{~Hz}$ and $g_{\text {in }}=2 \mathrm{~g}$ for various sampling frequencies $f_{\mathrm{s}}$. The SNR remains relatively constant from $f_{\mathrm{s}}=100$ to $500 \mathrm{~Hz}$, whereas the resolution deteriorates at low sampling frequency.
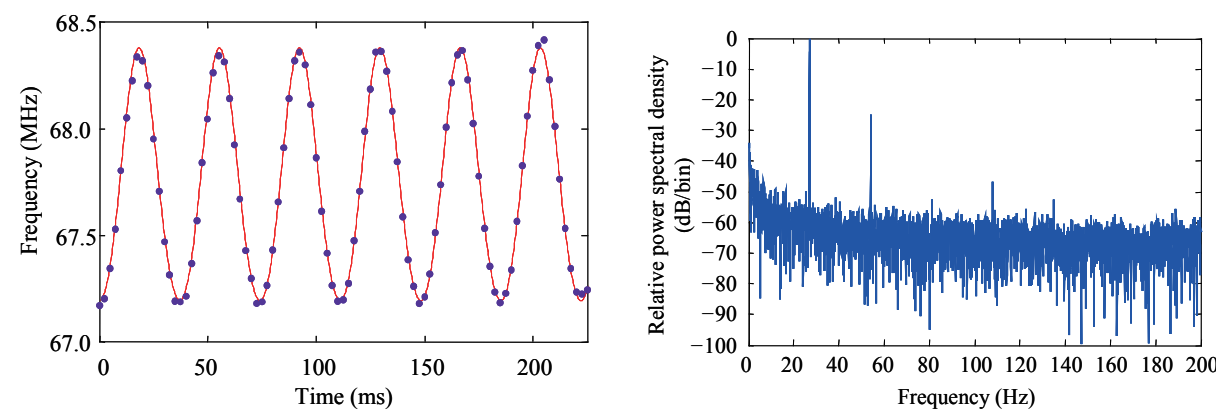

Fig. 14 (left). (Color online) Accelerometer output signal for $f_{\text {in }}=27 \mathrm{~Hz}, f_{\mathrm{s}}=400 \mathrm{~Hz}$, and $g_{\text {in }}=3 \mathrm{~g}$. Fig. 15 (right). (Color online) FFT spectrum of the signal in Fig. 14.

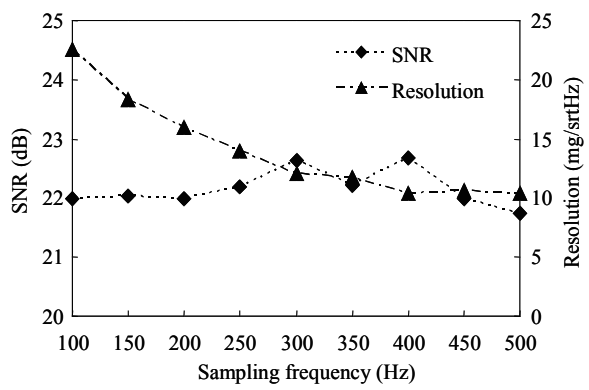

Fig. 16. Accelerometer performance at $f_{\text {in }}=27 \mathrm{~Hz}$ and $g_{\text {in }}=2 g$. 
This is caused by the large flicker noise shown in Fig. 15. As the sampling bandwidth is reduced, the flicker noise becomes more significant and thus increases the average noise floor and worsens the resolution. Figure 17 shows the sensor performance at $f_{\mathrm{s}}=400$ $\mathrm{Hz}$ and $g_{\mathrm{in}}=2 \mathrm{~g}$ for various shaker frequencies $f_{\mathrm{in}}$. Since the sampling frequency and sensor bandwidth are fixed, the SNR and resolution show a relatively flat response of the sensor within the input frequency range. Figure 18 shows the output amplitude at $f_{\text {in }}=$ $27 \mathrm{~Hz}$ and $f_{\mathrm{s}}=400 \mathrm{~Hz}$ for various input acceleration levels. The calculated absolute and relative sensitivities are $197 \mathrm{kHz} / g$ and $2.8 \times 10^{-3} \Delta f / f_{0} / g$, respectively, and the nonlinearity is $0.6 \%$. The measured sensitivities are about $58 \%$ and $78 \%$ of the theoretical estimation of $340 \mathrm{kHz} / g$ and $3.6 \times 10^{-3} \Delta f / f_{0} / g$, respectively, mainly due to the difference between the simulated and measured values of center oscillation frequency $f_{0}$ and mechanical resonance frequency $\omega_{\mathrm{m}}$. From eq. (5), the relative sensitivity $\Delta f l f_{0}$ is proportional to $\Delta R / R_{0}$, which in turn is proportional to the mechanical sensitivity $S_{\mathrm{m}}$, i.e., proof mass displacement per $g$. Since $S_{\mathrm{m}}$ is proportional to $1 / \omega_{\mathrm{m}}^{2}$, the reduction of measured $S_{\mathrm{m}}$ owing to the reduction of measured $\omega_{\mathrm{m}}$ is $(389 / 464)^{2}=70 \%$, close to the $78 \%$ reduction in measured $\Delta f l f_{0}$. The absolute sensitivity is proportional to $f_{0} \cdot S_{\mathrm{m}}$. Therefore, the reduction of absolute sensitivity calculated from the measured values is $(70.3 / 94) \cdot(389 / 464)^{2}$ $=53 \%$, close to the $58 \%$ reduction in measurement.

\section{Conclusions}

In this paper, we present a monolithic three-axis CMOS-MEMS accelerometer with single proof mass. The outputs of the accelerometer are converted to oscillation signals whose frequency shifts are proportional to the external acceleration. The accelerometer was fabricated by standard CMOS processes followed by backside and frontside dry etching postprocessing. The measured mechanical resonant frequency is $464 \mathrm{~Hz}$. The oscillation frequency of the $z$-axis oscillator is about $70 \mathrm{MHz}$. The measured absolute sensitivity, relative sensitivity, and resolution along the $z$-axis are $198 \mathrm{kHz} / \mathrm{g}, 2.8 \times 10^{-3}$ $\Delta f / f_{0} / g$, and $10.9 \mathrm{mG} / \sqrt{\mathrm{Hz}}$, respectively. The proposed accelerometer has achieved a much higher center frequency and sensitivity than the current accelerometers reported in the literature.
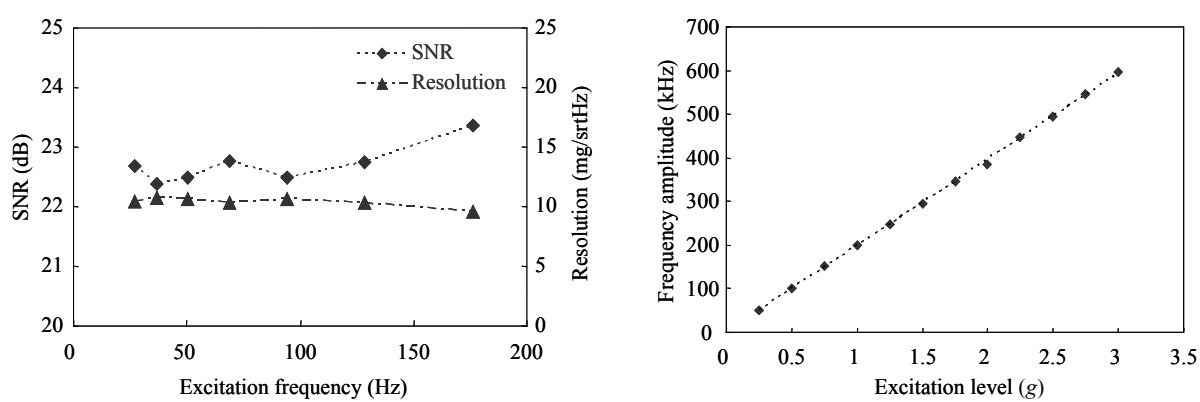

Fig. 17 (left). Accelerometer performance at $f_{\mathrm{s}}=400 \mathrm{~Hz}$ and $g_{\text {in }}=2 \mathrm{~g}$.

Fig. 18 (right). Accelerometer output amplitude at $f_{\text {in }}=27 \mathrm{~Hz}$ and $f_{\mathrm{s}}=400 \mathrm{~Hz}$. 


\section{Acknowledgements}

This work was supported in part by the National Science Council, Taiwan, R.O.C., under grant nos. NSC 101-2221-E-009-171 and NSC 101-2221-E-009-166. The authors thank the Chip Implementation Center, National Center for High-performance Computing, National Nano Device Laboratory, Nano Facility Center of National Chiao Tung University, and Center for Nanotechnology, Material Science, and Microsystem of National Tsing Hwa University, Taiwan, R.O.C., for the use of their facilities.

\section{References}

1 M. Lemkin and B. E. Boser: IEEE J. Solid-State Circuits 34 (1999) 456.

2 J. Wu, G. K. Fedder and L. R. Carley: IEEE J. Solid-State Circuits 39 (2004) 722.

3 C.-M. Sun, M.-H. Tsai, Y.-C. Liu and W. Fang: IEEE Trans. Electron Devices 57 (2010) 1670 .

4 F. Mailly, A. Giani, A. Martinez, R. Bonnot, P. Temple-Boyer and A. Boyer: Sens. Actuators, A 103 (2003) 359.

5 S. J. Chen and C. H. Shen: IEEE Trans. Instrum. Meas. 57 (2008) 1572.

6 A. Partridge, J. K. Reynolds, B. W. Chui, E. M. Chow, A. M. Fitzgerald, L. Zhang, N. Maluf and T. W. Kenny: J. Microelectromech. Syst. 9 (2000) 58.

7 H. Takao, H. Fukumoto and M. Ishida: IEEE Trans. Electron Devices 48 (2001) 1961.

8 P. Dong, X. Li, H. Yang, H. Bao, W. Zhou, S. Li and S. Feng: Sens. Actuators, A 141 (2008) 339.

9 M. Haris Md Khir, P. Qu and H. Qu: Sensors 11 (2011) 7892.

10 H.-S. Hsieh, H.-C. Chang, C.-F. Hu, C.-L. Cheng and W. Fang: J. Micromech. Microeng. 21 (2011) 105006.

11 J. Han, C. Zhu, J. Liu and P. Li: J. Micromech. Microeng. 15 (2005) 702.

12 E. J. Eklund and A. M. Shkel: J. Micromech. Microeng. 17 (2007) 730.

13 Z. Tang, S. Fan, W. Xing, Z. Guo and Z. Zhang: Microsyst. Technol. 17 (2011) 1481.

14 A. A. Seshia, M. Palaniapan, T. A. Roessig, R. T. Howe, R. W. Gooch, T. R. Schimert and S. Montague: J. Microelectromech. Syst. 11 (2002) 784.

15 C. Comi, A. Corigliano, G. Langfelder, A. Longoni, A. Tocchio and B. Simoni: J. Microelectromech. Syst. 19 (2010) 1140.

16 A. A. Trusov, S. A. Zotov, B. R. Simon and A. M. Shkel: Proc. IEEE MEMS 2013, p. 29.

17 C. Burrer, J. Esteve and E. Lora-Tamayo: J. Microelectromech. Syst. 5 (1996) 122.

18 M. Aikele, K. Bauer, W. Ficker, F. Neubauer, U. Prechtel, J. Schalk and H. Seidel: Sens Actuators, A 92 (2001) 161.

19 V. Ferrari, A. Ghisla, D. Marioli and A. Taroni: Sens. Actuators, A 123 (2005) 258.

20 Y.-T. Liao, W. J. Biederman and B. P. Otis: IEEE Sensors J. 11 (2011) 114.

21 Y. Chiu, H.-C. Hong and P.-C. Wu: Proc. IEEE Sensors 2012, p. 166.

22 H. Wang, Y. Chen, A. Hassibi, A. Scherer and A. Hajimiri: Proc. ISSCC 2009, p. 438.

23 S. Dalola, V. Ferrari and D. Marioli: Meas. Sci. Technol. 23 (2012) 035107.

24 L. B. Oliveira, A. Allam, I. M. Filanovsky, J. R. Fernandes, C. J. M. Verhoeven and M. M. Silva: Int. J. Circ. Theor. Appl. 38 (2010) 681. 